\title{
Vanishing heat conductivity limit for the 2D Cahn-Hilliard-Boussinesq system
}

\author{
Zaihong Jiang ${ }^{1 *}$ and Jishan Fan ${ }^{2}$
}

\footnotetext{
* Correspondence: jzhong@zjnu.cn ${ }^{1}$ Department of Mathematics, Zhejiang Normal University, Jinhua 321004, People's Republic of China Full list of author information is available at the end of the article
}

\section{Abstract}

This article studies the vanishing heat conductivity limit for the 2D Cahn-Hilliardboussinesq system in a bounded domain with non-slip boundary condition. The result has been proved globally in time. 2010 MSC: 35Q30; 76D03; 76D05; 76D07.

Keywords: Cahn-Hilliard-Boussinesq, inviscid limit, non-slip boundary condition

\section{Introduction}

Let $\Omega \subseteq \mathbb{R}^{2}$ be a bounded, simply connected domain with smooth boundary $\partial \Omega$, and $n$ is the unit outward normal vector to $\partial \Omega$. We consider the following Cahn-HilliardBoussinesq system in $\Omega \times(0, \infty)[1]$ :

$$
\begin{aligned}
& \partial_{t} u+(u \cdot \nabla) u+\nabla \pi-\Delta u=\mu \nabla \phi+\theta e_{2}, \\
& \operatorname{div} u=0, \\
& \partial_{t} \theta+u \cdot \nabla \theta=\varepsilon \Delta \theta, \\
& \partial_{t} \phi+u \cdot \nabla \phi=\Delta \mu, \\
& -\Delta \phi+f^{\prime}(\phi)=\mu, \\
& u=0, \theta=0, \frac{\partial \phi}{\partial n}=\frac{\partial \mu}{\partial n}=0 \quad \text { on } \quad \partial \Omega \times(0, \infty), \\
& (u, \theta, \phi)(x, 0)=\left(u_{0}, \theta_{0}, \phi_{0}\right)(x), x \in \Omega,
\end{aligned}
$$

where $u, \pi, \theta$ and $\varphi$ denote unknown velocity field, pressure scalar, temperature of the fluid and the order parameter, respectively. $\varepsilon>0$ is the heat conductivity coefficient and $e_{2}:=(0,1)^{t} . \mu$ is a chemical potential and $f(\phi):=\frac{1}{4}\left(\phi^{2}-1\right)^{2}$ is the double well potential.

When $\varphi=0$, (1.1), (1.2) and (1.3) is the well-known Boussinesq system. In [2] Zhou and Fan proved a regularity criterion $\omega \dot{=}$ curlu $\in L^{1}\left(0, T ; \dot{B}_{\infty, \infty}^{0}\right)$ for the 3D Boussinesq system with partial viscosity. Later, in [3] Zhou and Fan studied the Cauchy problem

(c) 2011 Jiang and Fan; licensee Springer. This is an Open Access article distributed under the terms of the Creative Commons Attribution License (http://creativecommons.org/licenses/by/2.0), which permits unrestricted use, distribution, and reproduction in any medium, provided the original work is properly cited. 
of certain Boussinesq- $\alpha$ equations in $n$ dimensions with $n=2$ or 3 . We establish regularity for the solution under $\nabla u \in L^{1}\left(0, T ; \dot{B}_{\infty, \infty}^{0}\right)$. Here $\dot{B}_{\infty, \infty}^{0}$ denotes the homogeneous Besov space. Chae [4] studied the vanishing viscosity limit $\varepsilon \rightarrow 0$ when $\Omega=\mathbb{R}^{2}$. The aim of this article is to prove a similar result. We will prove that

Theorem 1.1. Let $\left(u_{0}, \theta_{0}\right) \in H_{0}^{1} \cap H^{2}, \varphi_{0} \in H^{4}$, div $u_{0}=0$ in $\Omega$ and $\frac{\partial \phi_{0}}{\partial n}=\frac{\partial \mu_{0}}{\partial n}=0$ on $\partial \Omega$. Then, there exists a positive constant $C$ independent of $\varepsilon$ such that

$$
\begin{aligned}
\left\|u_{\varepsilon}\right\|_{L^{\infty}\left(0, T ; H^{2}\right)} \leq C,\left\|\theta_{\varepsilon}\right\|_{L^{\infty}\left(0, T ; H^{2}\right)} \leq C, \\
\left\|\phi_{\varepsilon}\right\|_{L^{\infty}\left(0, T ; H^{4}\right)} \leq C,\left\|\partial_{t}\left(u_{\epsilon}, \theta_{\varepsilon}, \phi_{\epsilon}\right)\right\|_{L^{2}\left(0, T ; L^{2}\right)} \leq C,
\end{aligned}
$$

for any $T>0$, which implies

$$
\left(u_{\varepsilon}, \theta_{\varepsilon}, \phi_{\varepsilon}\right) \rightarrow(u, \theta, \phi) \text { strongly in } L^{2}\left(0, T ; H^{1}\right) \text { when } \varepsilon \rightarrow 0 .
$$

Here, $(u, \theta, \varphi)$ is the solution of the problem (1.1)-(1.7) with $\varepsilon=0$.

\section{Proof of Theorem 1.1}

Since (1.9) follows easily from (1.8) by the Aubin-Lions compactness principle, we only need to prove the a priori estimates (1.8). From now on, we will drop the subscript $\varepsilon$ and throughout this section $C$ will be a constant independent of $\varepsilon$.

First, by the maximum principle, it follows from (1.2), (1.3), and (1.6) that

$$
\|\theta\|_{L^{\infty}\left(0, T ; L^{\infty}\right)} \leq\left\|\theta_{0}\right\|_{L^{\infty}} \leq C .
$$

Testing (1.3) by $\theta$, using (1.2) and (1.6), we see that

$$
\frac{1}{2} \frac{\mathrm{d}}{\mathrm{d} t} \int \theta^{2} \mathrm{~d} x+\varepsilon \int|\nabla \theta|^{2} \mathrm{~d} x=0
$$

whence

$$
\sqrt{\varepsilon}\|\theta\|_{L^{2}\left(0, T ; H^{1}\right)} \leq C .
$$

Testing (1.1) and (1.4) by $u$ and $\mu$, respectively, using (1.2), (1.6), (2.1), and summing up the result, we find that

$$
\begin{aligned}
& \frac{\mathrm{d}}{\mathrm{d} t} \int \frac{1}{2} u^{2}+\frac{1}{2}|\nabla \phi|^{2}+f(\phi) \mathrm{d} x+\int|\nabla u|^{2}+|\nabla \mu|^{2} \mathrm{~d} x \\
= & \int \theta e_{2} u \mathrm{~d} x \leq\|\theta\|_{L^{2}}\|u\|_{L^{2}} \leq C\|u\|_{L^{2},}
\end{aligned}
$$

which gives

$$
\begin{aligned}
& \|\phi\|_{L^{\infty}\left(0, T ; H^{1}\right)} \leq C, \\
& \|u\|_{L^{\infty}\left(0, T ; L^{2}\right)}+\|u\|_{L^{2}\left(0, T ; H^{1}\right)} \leq C, \\
& \|\nabla \mu\|_{L^{2}\left(0, T ; L^{2}\right)} \leq C .
\end{aligned}
$$

Testing (1.4) by $\varphi$, using (1.2), (1.5) and (1.6), we infer that

$$
\begin{aligned}
& \frac{1}{2} \frac{\mathrm{d}}{\mathrm{d} t} \int \phi^{2} \mathrm{~d} x+\int|\Delta \phi|^{2} \mathrm{~d} x=\int\left(\phi^{3}-\phi\right) \Delta \phi \mathrm{d} x \\
= & -3 \int \phi^{2}|\nabla \phi|^{2} \mathrm{~d} x-\int \phi \Delta \phi \mathrm{d} x \leq-\int \phi \Delta \phi \mathrm{d} x \\
\leq & \frac{1}{2} \int|\Delta \phi|^{2} \mathrm{~d} x+\frac{1}{2} \int \phi^{2} \mathrm{~d} x,
\end{aligned}
$$


which leads to

$$
\|\phi\|_{L^{2}\left(0, T ; H^{2}\right)} \leq C .
$$

We will use the following Gagliardo-Nirenberg inequality:

$$
\|\phi\|_{L^{\infty}}^{2} \leq C\|\phi\|_{L^{6}}\|\phi\|_{H^{2}} .
$$

It follows from (2.6), (2.7), (2.5), (2.3) and (1.5) that

$$
\begin{aligned}
& \int_{0}^{T} \int|\nabla \Delta \phi|^{2} \mathrm{~d} x \mathrm{~d} t \\
= & \int_{0}^{T} \int\left|\nabla\left(f^{\prime}(\phi)-\mu\right)\right|^{2} \mathrm{~d} x \mathrm{~d} t \\
\leq & C \int_{0}^{T} \int|\nabla \mu|^{2} \mathrm{~d} x \mathrm{~d} t+C \int_{0}^{T} \int\left|\nabla\left(\phi^{3}-\phi\right)\right|^{2} \mathrm{~d} x \mathrm{~d} t \\
\leq & C+C \int_{0}^{T} \int \phi^{4}|\nabla \phi|^{2} \mathrm{~d} x \mathrm{~d} t \\
\leq & C+C\|\nabla \phi\|_{L^{\infty}\left(0, T ; L^{2}\right)}^{2} \int_{0}^{T}\|\phi\|_{L^{\infty}}^{4} \mathrm{~d} t \\
\leq & C+C \int_{0}^{T}\|\phi\|_{L^{6}}^{2}\|\phi\|_{H^{2}}^{2} \mathrm{~d} t \\
\leq & C+C\|\phi\|_{L^{\infty}\left(0, T ; H^{1}\right)}^{2} \int_{0}^{T}\|\phi\|_{H^{2}}^{2} \mathrm{~d} t \leq C,
\end{aligned}
$$

which yields

$$
\begin{gathered}
\|\phi\|_{L^{2}\left(0, T ; H^{3}\right)} \leq C \\
\|\phi\|_{L^{4}\left(0, T ; L^{\infty}\right)} \leq C \\
\|\nabla \phi\|_{L^{2}\left(0, T ; L^{\infty}\right)} \leq C .
\end{gathered}
$$

Testing (1.4) by $\Delta^{2} \varphi$, using (1.5), (2.4), (2.3), (2.10) and (2.11), we derive

$$
\begin{aligned}
& \frac{1}{2} \frac{d}{\mathrm{~d} t} \int|\Delta \phi|^{2} \mathrm{~d} x+\int\left|\Delta^{2} \phi\right|^{2} \mathrm{~d} x \\
& =-\int u \cdot \nabla \phi \cdot \Delta^{2} \phi \mathrm{d} x+\int \Delta\left(\phi^{3}-\phi\right) \cdot \Delta^{2} \phi \mathrm{d} x \\
& \leq\|u\|_{L^{2}}\|\nabla \phi\|_{L^{\infty}}\left\|\Delta^{2} \phi\right\|_{L^{2}}+\left\|\Delta\left(\phi^{3}-\phi\right)\right\|_{L^{2}}\left\|\Delta^{2} \phi\right\|_{L^{2}} \\
& \leq C\|\nabla \phi\|_{L^{\infty}}\left\|\Delta^{2} \phi\right\|_{L^{2}} \\
& +C\left(\|\phi\|_{L^{\infty}}^{2}\|\Delta \phi\|_{L^{2}}+\|\phi\|_{L^{\infty}}\|\nabla \phi\|_{L^{\infty}}\|\nabla \phi\|_{L^{2}}+\|\Delta \phi\|_{L^{2}}\right)\left\|\Delta^{2} \phi\right\|_{L^{2}} \\
& \leq C\|\nabla \phi\|_{L^{\infty}}\left\|\Delta^{2} \phi\right\|_{L^{2}} \\
& +C\left(\|\phi\|_{L^{\infty}}^{2}\|\Delta \phi\|_{L^{2}}+\|\phi\|_{H^{2}}\|\nabla \phi\|_{L^{\infty}}+\|\Delta \phi\|_{L^{2}}\right)\left\|\Delta^{2} \phi\right\|_{L^{2}} \\
& \leq \frac{1}{2}\left\|\Delta^{2} \phi\right\|_{L^{2}}^{2}+C\|\nabla \phi\|_{L^{\infty}}^{2}+C\|\phi\|_{L^{\infty}}^{4}\|\Delta \phi\|_{L^{2}}^{2} \\
& +C\|\nabla \phi\|_{L^{\infty}}^{2}\|\phi\|_{H^{2}}^{2}+C\|\Delta \phi\|_{L^{2}}^{2}
\end{aligned}
$$


which implies

$$
\|\phi\|_{L^{\infty}\left(0, T ; H^{2}\right)}+\|\phi\|_{L^{2}\left(0, T ; H^{4}\right)} \leq C .
$$

Testing (1.1) by $-\Delta u+\nabla \pi$, using (1.2), (1.6), (2.12), (2.1) and (2.4), we reach

$$
\begin{aligned}
& \frac{1}{2} \frac{\mathrm{d}}{\mathrm{d} t} \int|\nabla u|^{2} \mathrm{~d} x+\int(-\Delta u+\nabla \pi)^{2} \mathrm{~d} x \\
& =\int\left(\mu \nabla \phi+\theta e_{2}-u \cdot \nabla u\right)(-\Delta u+\nabla \pi) \mathrm{d} x \\
& \leq\left(\|\mu\|_{L^{2}}\|\nabla \phi\|_{L^{\infty}}+\|\theta\|_{L^{2}}+\|u\|_{L^{4}}\|\nabla u\|_{L^{4}}\right)\|-\Delta u+\nabla \pi\|_{L^{2}} \\
& \leq C\left(\|\nabla \phi\|_{L^{\infty}}+1+\|u\|_{L^{2}}^{1 / 2}\|\nabla u\|_{L^{2}}^{1 / 2} \cdot\|\nabla u\|_{L^{2}}^{1 / 2}\|\Delta u\|_{L^{2}}^{1 / 2}\right)\|-\Delta u+\nabla \pi\|_{L^{2}} \\
& \leq C\|\nabla \phi\|_{L^{\infty}}^{2}+C+C\|\nabla u\|_{L^{2}}^{4}+\frac{1}{2}\|-\Delta u+\nabla \pi\|_{L^{2}}^{2}
\end{aligned}
$$

which yields

$$
\|u\|_{L^{\infty}\left(0, T ; H^{1}\right)}+\|u\|_{L^{2}\left(0, T ; H^{2}\right)} \leq C .
$$

Here, we have used the Gagliardo-Nirenberg inequalities:

$$
\begin{aligned}
& \|u\|_{L^{4}}^{2} \leq C\|u\|_{L^{2}}\|\nabla u\|_{L^{2}}, \\
& \|\nabla u\|_{L^{4}}^{2} \leq C\|\nabla u\|_{L^{2}}\|u\|_{H^{2}},
\end{aligned}
$$

and the $H^{2}$-theory of the Stokes system:

$$
\|u\|_{H^{2}}+\|\pi\|_{H^{1}} \leq C\|-\Delta u+\nabla \pi\|_{L^{2}} .
$$

Similarly to (2.13), we have

$$
\left\|\partial_{t} u\right\|_{L^{2}\left(0, T ; L^{2}\right)} \leq C \text {. }
$$

(1.1), (1.2), (1.6) and (1.7) can be rewritten as

$$
\left\{\begin{array}{l}
\partial_{t} u-\Delta u+\nabla \pi=g:=\mu \nabla \phi+\theta e_{2}-u \cdot \nabla u, \text { in } \Omega \times(0, \infty) \\
u=0, \text { on } \partial \Omega \times(0, \infty) \\
u(x, 0)=u_{0}(x)
\end{array}\right.
$$

Using (2.12), (2.1), (2.13), and the regularity theory of Stokes system, we have

$$
\begin{aligned}
& \left\|\partial_{t} u\right\|_{L^{2}\left(0, T ; L^{p}\right)}+\|u\|_{L^{2}\left(0, T ; W^{2, p}\right)} \leq C\|g\|_{L^{2}\left(0, T ; L^{p}\right)} \\
\leq & C\|\mu\|_{L^{2}\left(0, T ; L^{\infty}\right)}\|\nabla \phi\|_{L^{\infty}\left(0, T ; L^{p}\right)}+C\|\theta\|_{L^{\infty}\left(0, T ; L^{\infty}\right)} \\
& +C\|u\|_{L^{\infty}\left(0, T ; L^{2 p}\right)}\|\nabla u\|_{L^{2}\left(0, T ; L^{2 p}\right)} \leq C
\end{aligned}
$$

for any $2<p<\infty$.

(2.16) gives

$$
\|\nabla u\|_{L^{2}\left(0, T ; L^{\infty}\right)} \leq C
$$

It follows from (1.3) and (1.6) that

$$
\Delta \theta=0 \text { on } \partial \Omega \times(0, \infty) .
$$

Applying $\Delta$ to (1.3), testing by $\Delta \theta$, using (1.2), (1.6), (2.16), (2.17) and (2.18), we obtain 


$$
\begin{aligned}
& \frac{1}{2} \frac{\mathrm{d}}{\mathrm{d} t} \int|\Delta \theta|^{2} \mathrm{~d} x+\varepsilon \int|\nabla \Delta \theta|^{2} \mathrm{~d} x \\
= & -\int(\Delta(u \cdot \nabla \theta)-u \nabla \Delta \theta) \Delta \theta \mathrm{d} x \\
\leq & C\left(\|\Delta u\|_{L^{4}}\|\nabla \theta\|_{\left.L^{4}+\|\nabla u\|_{L^{\infty}}\|\Delta \theta\|_{L^{2}}\right)\|\Delta \theta\|_{L^{2}}} \leq C\left(\|\Delta u\|_{L^{4}}+\|\nabla u\|_{L^{\infty}}\right)\|\Delta \theta\|_{L^{2}}^{2}\right.
\end{aligned}
$$

which implies

$$
\|\theta\|_{L^{\infty}\left(0, T ; H^{2}\right)}+\sqrt{\varepsilon}\|\theta\|_{L^{2}\left(0, T ; H^{3}\right)} \leq C .
$$

It follows from (1.3), (1.6), (2.19) and (2.13) that

$$
\left\|\partial_{t} \theta\right\|_{L^{\infty}\left(0, T ; L^{2}\right)} \leq C
$$

Taking $\partial_{t}$ to (1.4) and (1.5), testing by $\partial_{t} \varphi$, using (1.2), (1.6), (2.12), and (2.15), we have

$$
\begin{aligned}
& \frac{1}{2} \frac{\mathrm{d}}{\mathrm{d} t} \int\left|\partial_{t} \phi\right|^{2} \mathrm{~d} x+\int\left|\Delta \partial_{t} \phi\right|^{2} \mathrm{~d} x \\
= & -\int \partial_{t} u \cdot \nabla \phi \cdot \partial_{t} \phi \mathrm{d} x+\int \Delta\left(3 \phi^{2} \partial_{t} \phi-\partial_{t} \phi\right) \cdot \partial_{t} \phi \mathrm{d} x \\
= & -\int \partial_{t} u \cdot \nabla \phi \cdot \partial_{t} \phi \mathrm{d} x+\int\left(3 \phi^{2} \partial_{t} \phi-\partial_{t} \phi\right) \Delta \partial_{t} \phi \mathrm{d} x \\
\leq & \left\|\partial_{t} u\right\|_{L^{2}}\|\nabla \phi\|_{L^{\infty}}\left\|\partial_{t} \phi\right\|_{L^{2}}+\left(\|3 \phi\|_{L^{\infty}}^{2}+1\right)\left\|\partial_{t} \phi\right\|_{L^{2}}\left\|\Delta \partial_{t} \phi\right\|_{L^{2}} \\
\leq & \left\|\partial_{t} u\right\|_{L^{2}}\|\nabla \phi\|_{L^{\infty}}\left\|\partial_{t} \phi\right\|_{L^{2}}+\frac{1}{2}\left\|\Delta \partial_{t} \phi\right\|_{L^{2}}^{2}+C\left\|\partial_{t} \phi\right\|_{L^{2}}^{2}
\end{aligned}
$$

which gives

$$
\left\|\partial_{t} \phi\right\|_{L^{\infty}\left(0, T ; L^{2}\right)}+\left\|\partial_{t} \phi\right\|_{L^{2}\left(0, T ; H^{2}\right)} \leq C .
$$

By the regularity theory of elliptic equation, it follows from (1.4), (1.5), (1.6), (2.21), (2.13) and (2.12) that

$$
\begin{aligned}
\|\phi\|_{L^{\infty}\left(0, T ; H^{4}\right)} \leq & C\|\Delta\|_{L^{\infty}\left(0, T ; H^{2}\right)} \leq C\left\|\mu-f^{\prime}(\phi)\right\|_{L^{\infty}\left(0, T ; H^{2}\right)} \\
& \leq C\|\mu\|_{L^{\infty}\left(0, T ; H^{2}\right)}+C\left\|f^{\prime}(\phi)\right\|_{L^{\infty}\left(0, T ; H^{2}\right)} \\
& \leq C\|\Delta \mu\|_{L^{\infty}\left(0, T ; L^{2}\right)}+C\left\|f^{\prime}(\phi)\right\|_{L^{\infty}\left(0, T ; H^{2}\right)} \\
& \leq C\left\|\partial_{t} \phi+u \cdot \nabla \phi\right\|_{L^{\infty}\left(0, T ; L^{2}\right)}+C\left\|f^{\prime}(\phi)\right\|_{L^{\infty}\left(0, T ; H^{2}\right)} \\
& \leq C\left\|\partial_{t} \phi\right\|_{L^{\infty}\left(0, T ; L^{2}\right)}+C\|u\|_{L^{\infty}\left(0, T ; L^{4}\right)}\|\nabla \phi\|_{L^{\infty}\left(0, T ; L^{4}\right)} \\
& +C\left\|f^{\prime}(\phi)\right\|_{L^{\infty}\left(0, T ; H^{2}\right)} \leq C .
\end{aligned}
$$

Taking $\partial_{t}$ to (1.1), testing by $\partial_{t} u$, using (1.2), (1.6), (2.17), (2.22), (2.21) and (1.5), we conclude that

$$
\begin{aligned}
& \frac{1}{2} \frac{\mathrm{d}}{\mathrm{d} t} \int\left|\partial_{t} u\right|^{2} \mathrm{~d} x+\int\left|\nabla \partial_{t} u\right|^{2} \mathrm{~d} x \\
= & -\int \partial_{t} u \cdot \nabla u \cdot \partial_{t} u \mathrm{~d} x+\int\left(\partial_{t} \mu \cdot \nabla \phi+\mu \cdot \nabla \partial_{t} \phi+\partial_{t} \theta e_{2}\right) \partial_{t} u \mathrm{~d} x \\
\leq & \|\nabla u\|_{L^{\infty}}\left\|\partial_{t} u\right\|_{L^{2}}^{2}+\left(\left\|\partial_{t} u\right\|_{L^{2}}\|\nabla \phi\|_{L^{\infty}}+\|\mu\|_{L^{\infty}}\left\|\nabla \partial_{t} \phi\right\|_{L^{2}}+\left\|\partial_{t} \theta\right\|_{L^{2}}\right)\left\|\partial_{t} u\right\|_{L^{2}} \\
\leq & \|\nabla u\|_{L^{\infty}}\left\|\partial_{t} u\right\|_{L^{2}}^{2}+C\left(\left\|\Delta \partial_{t} \phi\right\|_{L^{2}}+\left\|\partial_{t}\left(\phi^{3}-\phi\right)\right\|_{L^{2}}+\left\|\nabla \partial_{t} \phi\right\|_{L^{2}}+1\right)\left\|\partial_{t} u\right\|_{L^{2}}
\end{aligned}
$$


which implies

$$
\left\|\partial_{t} u\right\|_{L^{\infty}\left(0, T ; L^{2}\right)}+\left\|\partial_{t} u\right\|_{L^{2}\left(0, T ; H^{1}\right)} \leq C .
$$

Using (2.23), (2.22), (2.1), (2.13), (1.1), (1.2), (1.6) and the $H^{2}$-theory of the Stokes system, we arrive at

$$
\|u\|_{L^{\infty}\left(0, T ; H^{2}\right)} \leq C .
$$

This completes the proof.

\section{Acknowledgements}

This study was supported by the NSFC (No. 11171154) and NSFC (Grant No. 11101376).

\section{Author details}

'Department of Mathematics, Zhejiang Normal University, Jinhua 321004, People's Republic of China ${ }^{2}$ Department of Applied Mathematics, Nanjing Forestry University, Nanjing 210037, People's Republic of China

\section{Authors' contributions}

All authors read and approved the final manuscript.

\section{Competing interests}

The authors declare that they have no competing interests.

Received: 18 October 2011 Accepted: 22 December 2011 Published: 22 December 2011

\section{References}

1. Boyer, Franck: Mathematical study of multi-phase flow under shear through order parameter formulation. Asymptot Anal. 20, 175-212 (1999)

2. Fan, Jishan, Zhou, Yong: A note on regularity criterion for the 3D Boussinesq system with partial viscosity. Appl Math Lett. 22, 802-805 (2009). doi:10.1016/j.aml.2008.06.041

3. Zhou, Yong, Fan, Jishan: On the Cauchy problems for certain Boussinesq-a equations. Proc R Soc Edinburgh Sect A. 140, 319-327 (2010). doi:10.1017/S0308210509000122

4. Chae, Dongho: Global regularity for the 2D Boussinesq equations with partial viscosity terms. Adv Math. 203, 497-513 (2006). doi:10.1016/j.aim.2005.05.001

doi:10.1186/1687-2770-2011-54

Cite this article as: Jiang and Fan: Vanishing heat conductivity limit for the 2D Cahn-Hilliard-Boussinesq system. Boundary Value Problems 2011 2011:54.

\section{Submit your manuscript to a SpringerOpen ${ }^{\circ}$} journal and benefit from:

- Convenient online submission

Rigorous peer review

- Immediate publication on acceptance

- Open access: articles freely available online

- High visibility within the field

- Retaining the copyright to your article

Submit your next manuscript at $>$ springeropen.com 